\title{
A NEW CATALOGUE OF FAINT GALAXIES
}

\author{
Leopoldo Infante \\ Department of Physics and Astronomy, University of Victoria \\ Astrophysics Group, Pontificia Universidad Catolica de Chile \\ Casilla 6014, Santiago, Chile
}

\begin{abstract}
A new catalogue of faint galaxies on a $3 \mathrm{deg}^{2}$ area near the North Galactic Pole (NGP), complete to $J=24.5$ and $F=23.5$, has been obtained from nine Canada France Hawaii Telescope prime focus plates. The angular correlation function has been measured for the first time to separations $\sim 1^{\circ}$. Preliminary results indicate a power law slope of $\approx-0.8$ at small separations and no sharp break for $\theta \leq 1^{\circ}$. We also find some evidence that the power law slope flattens to fainter limiting magnitudes.
\end{abstract}

Further progress in the study of galaxy clustering demands deep samples (i.e., $J \leq 23$ ) over extended areas of the sky $(\theta \geq 0$. 5). The combination of a faint limiting magnitude $(J \approx 24.5)$ and a large angular extent $\left(3^{\circ}\right)$ samples a volume similar to that of the existing shallow surveys $\left(\sim 10^{6} \mathrm{Mpc}\right)$ at a large look-back time $(z \sim 0.7)$. Two important goals of the project are (a) to study the angular correlation function for very deep samples of galaxies at large angular separations and (b) to obtain $\omega(\theta)$ as a function of morphological type or color for deep samples of galaxies. We report preliminary results on our first goal.

In April 1987 the CFHT $3.6 \mathrm{~m}$ telescope was used to obtain nine plates in $J$ and $F$ of five fields near the NGP. The fields were selected to minimize galactic obscuration and to maximize the angular extension of the survey. The total area covered by the plates is $4 \mathrm{deg}^{2}$, of which $2.7 \mathrm{deg}^{2}$ were digitized and pre-reduced with the University of Cambridge APM machine (Kibblewhite et al. 1983).

For the project to succeed, proper calibration of the data is essential. The following steps were taken: (a) Simulations were performed to choose the best configuration on the sky. See Figure 1. (b) To transform instrumental APM isophotal magnitudes to linear total (á la Kron 1980; Irwin and Hall 1982) magnitudes, the center $4000 \times 4000$ pixel of each area was raster scanned, linearized, and analysed with a total algorithm. (c) Standard sequences were observed with CCD at the $0.9 \mathrm{~m}$ Kitt Peak National Observatory in $B$ and $R$ on five locations in each field to monitor zero-point variation. The errors in the determination of these values is $\leq 0.05$ mag. (d) Star/Galaxy classification was performed with the APM standard methods. (e) To transform machine coordinates to celestial coordinates, we used secondary astrometric standards obtained from a red Palomar Observatory Sky Survey plate (O1393). (f) A variety of images and bad areas were removed from the catalogue.

The final catalogue contains nearly 70,000 galaxies in $J\left(2.7 \mathrm{deg}^{2}\right)$ and 60,000 galaxies in $F$ (2.1 $\mathrm{deg}^{2}$ ). Information about positions, magnitudes, image moments, areal profiles, sizes, and colours is available for stars, galaxies, mergers, and peculiar images. The catalogue appears to be $95 \%$ complete at $J=24.5$ and $F=23.5$. Magnitude errors range from \pm 0.5 at $J=24.5$ to $\leq \pm 0.1$ at $J=20$. We plan to store this catalogue in an optical disk and it will be available upon request in the near future.

To check the validity of the catalogue we performed galaxy counts. We obtained slopes consistent with previous results (Tyson 1988; Koo 1986; Infante, et al. 1986), 0.45 for $J$ catalogue and 0.35 for the $F$ catalogue. Although the zero points of our counts are lower than in Koo (1986) (SA57, same area), our results are in almost perfect agreement with the CCD galaxy count results (Tyson 1988). No evidence for large field-to-field variations $(\leq 5 \%)$ is present in our catalogues.

We have estimated the angular correlation function $\omega(\theta)$ using methods described in Pritchet and Infante (1976) and in Koo and Szalay (1984). We calculated $\omega(\theta)$ for each indivi- 

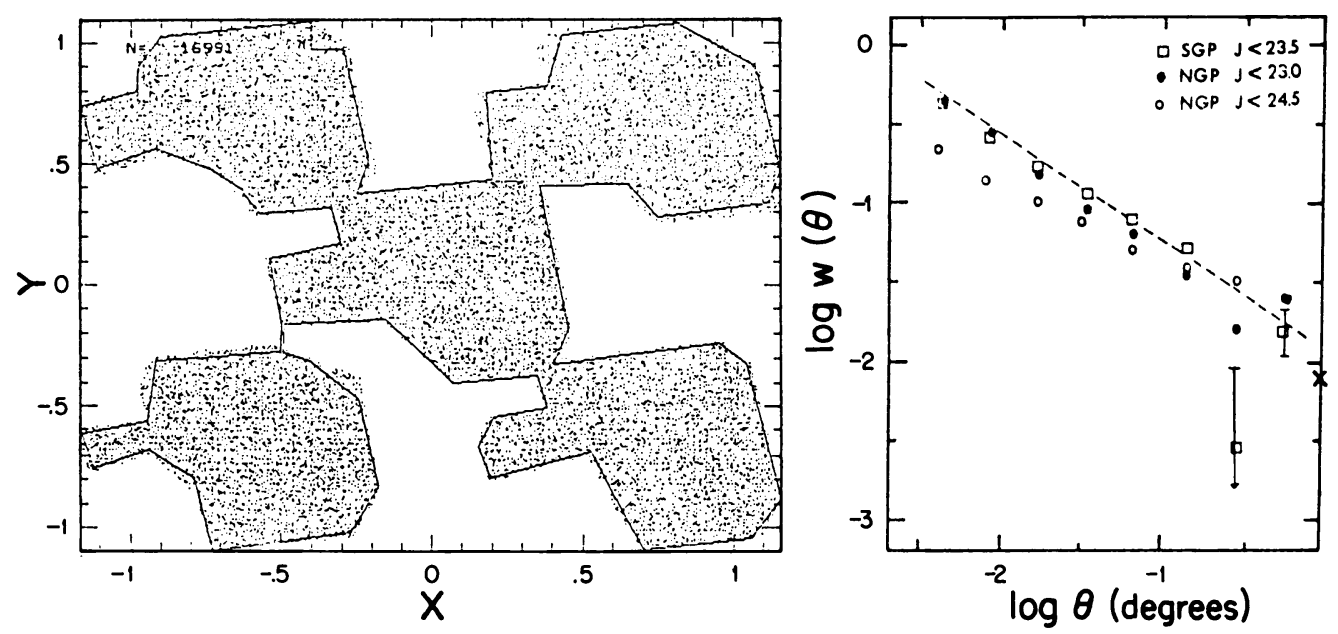

Figure 1. The distribution of galaxies $(J \leq 23$ and $F \leq 21.5)$ for the $2^{\circ} \times 2^{\circ}$ NGP catalogue. $X$ and $Y$ are in degrees from the center. Only points inside the contour are included.

Figure 2. Angular correlation function as a function of limiting magnitude. The line has a slope of -0.8 . Note the $x$, obtained in a separate run for large separations. In the cases where no error bars are drawn, the poison errors are smaller than the symbols.

dual field at small separations $(\theta \leq 0.6)$ and to a limiting $J$ magnitude of 24.5. After fitting a power law of form $\omega(\theta)=A \theta^{-\delta}$ to each sample, we found that $\delta=0.8 \pm 0.1$. By forcing this index in the range $0.001^{\circ} \leq \theta \leq 0.1$ ), we found that $B$ varied from 0.985 to 0.995 . Figure 2 shows the combined result of $\omega(\theta)$ at small $\theta$. Previous estimates by Pritchet and Infante (1986) on a south galactic pole (SGP) field are also shown in this figure. At fainter magnitude the slope decreased significantly, perhaps due to evolution of the clustering pattern at large look-back times. We find no break in $\omega(\theta)$ at $\theta \approx 0.6$, as in the SGP.

Last-minute computations of $\omega(\theta)$ at large separations resulted in the cross $(x)$ in Figure 2 at $\theta=1^{\circ}$. These were performed for a catalogue that is complete to $J \leq 23$. No sharp break is detected for $\theta \leq 1^{\circ}$. The point that lies above the natural trend may be due to extra power in $\omega(\theta)$ introduced by the plate size. Note that the linear scale size of the fields is of the order of $\theta \approx 0.6$. Simulations á la Soneira and Peebles are carried out in order to detect any effects that the shape of the field configuration might introduce in the estimate of the $\omega(\theta)$.

Acknowledgments - I would like to thank the CFH telescope coorporation for granting six nights for this project, KPNO for the CCD observations, the Cambridge APM group for allowing me to use their scanner, Dr. C. J. Pritchet for continous support during the time of this research, and the University of Victoria for a pre-doctoral fellowship. Part of this research was carried out while visiting CTIO. This research was partially funded by FONDECYT Project \#89/0617.

\section{REFERENCES}

Infante, L., Pritchet, C.J., and Quintana, H. 1986, Astron. J., 91, 217.

Irwin, M.J., and Hall, P. 1982, Workshop Astronomical Measuring Machines, ed. Stobie and McInnes.

Kibblewhite, E.J., Bridgeland, M.T., Bunclark, P.S., and Irwin, M.J., 1983, Proc. Astronomical Microdensitometry Conference, Washington.

Koo, D.C., 1986, Ap. J. 311, 651.

Koo, D.C., and Szalay, A.S. 1984, Ap.J. 282, 390.

Kron, R.G., 1980, Ap. J. 43, 305.

Pritchet, C.J., and Infante, L. 1986, Astron. J., 91, 1.

Tyson, J.A. 1988, Astron. J., 96, 1. 